\title{
A double-blind randomised controlled trial testing the effect of a barley product containing varying amounts and types of fibre on the postprandial glucose response of healthy volunteers
}

\author{
Nancy Ames ${ }^{1,2 *} \dagger$, Heather Blewett ${ }^{2,3,4} \dagger$, Joanne Storsley ${ }^{1}$, Sijo J. Thandapilly ${ }^{1,2}$, Peter Zahradka ${ }^{2,3,4}$ and \\ Carla Taylor $2,3,4$ \\ ${ }^{1}$ Agriculture and Agri-Food Canada, Richardson Centre for Functional Foods and Nutraceuticals, 196 Innovation Drive, \\ Winnipeg, MB, Canada R3T 6C5 \\ ${ }^{2}$ Department of Human Nutritional Sciences, University of Manitoba, W383 Duff Roblin Building, Winnipeg, MB, \\ Canada R3T 2N2 \\ ${ }^{3}$ Canadian Centre for Agri-Food Research in Health and Medicine, St Boniface Hospital Research Centre, 351 Taché Avenue, \\ Winnipeg, MB, Canada R2H $2 A 6$ \\ ${ }^{4}$ Department of Physiology and Pathophysiology, University of Manitoba, 432 Basic Medical Sciences Building, \\ 745 Bannatyne Avenue, Winnipeg, MB, Canada R3E OJ9
}

(Submitted 18 September 2014 - Final revision received 9 January 2015 - Accepted 21 January 2015 - First published online 8 April 2015)

\section{Abstract}

The aim of the present study was to determine if the consumption of barley tortillas varying in fibre and/or starch composition affected postprandial glucose, insulin, glucagon-like peptide-1 (GLP-1) or peptide YY concentrations. A double-blind, randomised, controlled trial was performed with twelve healthy adults. They each consumed one of five barley tortillas or a glucose drink on six individual visits separated by at least 1 week. Tortillas were made from $100 \%$ barley flour blends using five different milling fractions to achieve the desired compositions. All treatments provided $50 \mathrm{~g}$ of available carbohydrate and were designed to make the following comparisons: (1) low-starch amylose $(0 \%) v$. high-starch amylose $(42 \%)$ with similar $\beta$-glucan and insoluble fibre content; (2) low $\beta$-glucan ( $4.5 \mathrm{~g}) v$. medium $\beta$-glucan $(7.8 \mathrm{~g}) v$. high $\beta$-glucan $(11.6 \mathrm{~g})$ with similar starch amylose and insoluble fibre content; and (3) low insoluble fibre ( $7.4 \mathrm{~g}) v$. high insoluble fibre $(19.6 \mathrm{~g})$ with similar starch amylose and $\beta$-glucan content. Blood was collected at fasting and at multiple intervals until 180 min after the first bite/sip of the test product. Amylose and insoluble fibre content did not alter postprandial glucose and insulin, but high- $\beta$-glucan tortillas elicited a lower glucose and insulin response as compared to the low- $\beta$-glucan tortillas. The tortillas with high insoluble fibre had a higher AUC for GLP-1 as compared to the tortillas with low insoluble fibre, whereas amylose and $\beta$-glucan content had no effect. Results show that processing methods can be used to optimise barley foods to reduce postprandial blood glucose responses and factors that may influence satiety.

Key words: Amylose: Barley: $\beta$-Glucan: Insoluble fibre: Postprandial glucose response

Barley is a high-fibre cereal grain that contains significant levels of both $\beta$-glucan and insoluble fibre, and it has been classified as having a low glycaemic index (GI). Health professionals recommend choosing foods that elicit a low postprandial glycaemic response (PPGR), without a concomitant increase in insulin, to improve glycaemic control ${ }^{(1)}$. Furthermore, the potential for the utilisation of barley in functional foods is high ${ }^{(2)}$. Barley has been evaluated for incorporation into several food products, including bread $^{(3-5)}$, pasta $^{(6)}$, biscuits $^{(7)}$, noodles ${ }^{(8)}$, tortillas and chips $^{(2,9)}$. Governmentapproved health claims have focused on the effect of the soluble fibre ( $\beta$-glucan) content of barley on $\operatorname{PPGR}^{(10)}$, but there are several factors that could be manipulated in barley foods to optimise PPGR, including the amount and type of fibre and the type of starch.

Barley starch, like that of other cereal grains, contains two forms of starch, amylose and amylopectin, each with specific physico-chemical properties. Amylose is a starch that has a

Abbreviations: BF-BG, bran flour with high $\beta$-glucan; BF-IDF, bran flour with high insoluble dietary fibre; GI, glycaemic index; GIT, gastrointestinal tract; GLP-1, glucagon-like peptide-1; HA-DFF, high-amylose dusted flour fractions; iAUC, incremental AUC; IDF, insoluble dietary fibre; PPGR, postprandial glycaemic response; PYY, peptide YY; SGF, straight-grade flour; WGF, wholegrain flour.

*Corresponding author: N. Ames, fax +1 204474 7552, email nancy.ames@agr.gc.ca

† Both of these authors contributed equally to the present work 
tightly packed structure, which slows and sometimes (in the case of resistant starch formed from retrograded amylose) prevents digestive enzymes from breaking it down to glucose. Amylopectin is a starch that has a branched structure and is easier to digest ${ }^{(11)}$. Numerous studies have shown that meals made with high-amylose or high-resistant starches (rice, maize and wheat) elicit a lower PPGR than meals made from high-amylopectin starches ${ }^{(11-14)}$. Therefore, it is plausible that a barley variety with a significantly higher amylose: amylopectin ratio would result in a food product with a lower PPGR. However, a study on plasma glucose and insulin after the consumption of barley varying in amylose content (7-44\% amylose) showed marginal differences in glycaemic response ${ }^{(15)}$, which suggests that other constituents, such as fibre, might play a role. By choosing a barley food with higher fibre levels, one would anticipate a blunted $\mathrm{PPGR}^{(16-18)}$. However, fibre can be classified as soluble or insoluble. $\beta$-Glucan is a soluble fibre which has been shown to lower PPGR. Numerous clinical trials have demonstrated that oat and barley foods containing $\beta$-glucan can lower blood glucose levels through several different mechanisms in both the upper gastrointestinal tract (GIT) and the lower intestinal tract ${ }^{(19)}$. In the upper GIT, soluble $\beta$-glucan can develop a high viscosity, thereby slowing the mixing of the meal with digestive enzymes ${ }^{(20)}$ and slowing the rate of gastric emptying $^{(21-23)}$. Insoluble dietary fibre (IDF), which includes resistant starch, like soluble dietary fibre, cannot be digested into glucose, so it is unclear whether the total fibre or the type of fibre has a greater effect on postprandial glucose levels. Barley fibre composition is significantly influenced by variety to a greater extent than other cereal grains are ${ }^{(24)}$ Large variation in fibre composition has been reported for barley genotypes from different origins, with the levels of total dietary fibre in wholegrain ranging from as low as $9 \%$ to greater than $30 \%{ }^{(25-30)}$. Similarly, a wide range of $\beta$-glucan content has been reported for barley $(3-15 \%)^{(25-28,30,31)}$, with Canadian hull-less varieties typically containing $4-9 \% \beta$-glucan ${ }^{(32,33)}$. It is also possible to further manipulate the fibre composition of barley through milling ${ }^{(2,5,34-36)}$. The physiological response to food products made with specific barley cultivars that have undergone optimised milling and processing treatments is unknown; this knowledge is critical in determining whether or not these optimisation treatments will have a beneficial influence on glycaemia and whether they are superior from a nutritional perspective.

The present study investigated the use of cultivar selection and milling fractions to formulate $100 \%$ barley flour tortillas with varying proportions of amylose/amylopectin, resistant starch, soluble fibre and insoluble fibre. Subsequently, a single-site, double-blind, randomised, controlled clinical trial was carried out with the objective of better understanding the relationship between the consumption of barley tortillas with varying starch and fibre compositions and PPGR while keeping available carbohydrate constant. Hormones that play a role in PPGR and satiety, such as the incretin glucagonlike peptide-1 (GLP-1) and the satiety hormone peptide YY (PYY), were also measured.

\section{Experimental methods}

\section{Barley samples and compositional analysis}

Grain samples of four hull-less barley genotypes were obtained from the Crop Development Centre, University of Saskatchewan, including two waxy, low-amylose varieties (CDC Candle, CDC Fibar) and two high-amylose types (SB94893, SH99250). Starch amylose content was confirmed according to a method developed by Schoch ${ }^{(37)}$. Grain was milled into wholegrain flour (WGF) and several fractions, including straight-grade flour (SGF), 'dusted flour from shorts', 'dusted flour from bran', 'concentrated shorts', 'concentrated bran' and 'pearlings', as described previously by Harding et $a l .{ }^{(38)}$. A commercial barley fraction with concentrated $\beta$-glucan content was obtained from PolyCell Technologies. The composition of the flour fractions was analysed using American Association of Cereal Chemists (AACC) International Approved Methods for insoluble and total dietary fibre (method 32-07.01 ${ }^{(39)}$ ), $\beta$-glucan (method 32-23.01 ${ }^{(40)}$ ), total starch (method 76-13.01 ${ }^{(41)}$ ) and resistant starch (method 32-40.01(42) ) as well as available carbohydrate content ${ }^{(43)}$. Based on the composition results (Table 1), CDC Fibar was selected for use as the low-amylose barley ( $0 \%$ amylose in its starch), because it had the highest fibre levels. Barley genotype SH99250 was selected for use as the high-amylose variety ( $42 \%$ starch amylose) (Table 1). Starch was extracted from the whole grain of each cultivar, and its amylose content was measured in order to confirm the amylose content of the different genotypes. High-amylose barley cultivars have been shown to have increased levels of dietary fibre because they have higher levels of resistant starch after heat processing ${ }^{(44)}$, which may result in a lower glycaemic response. Therefore, it was hypothesised that SH99250 as a high-amylose cultivar would contain higher resistant starch levels after being cooked into tortillas, would result in a low glycaemic response and would consequently have the potential for industrial significance in the future.

\section{Preparation of barley flour blends}

By varying the proportions of different milling fractions and making small adjustments to the serving sizes, several flour blend scenarios were created and examined for possible use in test meal treatments (Table 2). We selected five scenarios to supply a consistent $50 \mathrm{~g}$ of available carbohydrate per serving while varying the following starch/fibre levels: (1) low amylose (0\%) $v$. high amylose (42\%) with similar $\beta$-glucan and insoluble fibre content; (2) low $\beta$-glucan $(4.5 \mathrm{~g}) v$. medium $\beta$-glucan $(7.8 \mathrm{~g}) v$. high $\beta$-glucan $(11.6 \mathrm{~g})$ with similar amylose and insoluble fibre content; (3) low insoluble fibre $(7.4 \mathrm{~g}) v$. high insoluble fibre $(19.6 \mathrm{~g})$ with similar amylose and $\beta$-glucan content. All barley test meal formulations had more than $4 \mathrm{~g}$ of $\beta$-glucan per serving (Table 2). The high-amylose variety SH99250 was used for the highamylose dusted flour fractions (HA-DFF) flour blend; it was prepared by combining dusted flour from bran (37.7\%) and dusted flour from shorts (62.3\%). All other flour blends (WGF, SGF, bran flour with high $\beta$-glucan (BF-BG) and bran flour with high IDF (BF-IDF)) were prepared from the 
Table 1. Content of various carbohydrates in milling fractions from four barley genotypes

\begin{tabular}{|c|c|c|c|c|c|c|}
\hline Genotype & $\begin{array}{l}\text { Milling } \\
\text { fraction }\end{array}$ & $\begin{array}{l}\text { Total dietary } \\
\text { fibre }(\%, d b)\end{array}$ & $\begin{array}{l}\beta \text {-Glucan } \\
(\%, d b)\end{array}$ & $\begin{array}{c}\text { Available } \\
\text { carbohydrates } \\
(\%, \mathrm{db})\end{array}$ & $\begin{array}{l}\text { Total } \\
\text { starch } \\
(\%, d b)\end{array}$ & $\begin{array}{c}\text { Resistant } \\
\text { starch } \\
(\%, \mathrm{db})\end{array}$ \\
\hline \multirow{6}{*}{ SB94893 (starch amylose content $=41.48 \%$ ) } & WM & 17.45 & 7.68 & 64.14 & $53 \cdot 20$ & 1.63 \\
\hline & SGF & 12.09 & 4.91 & $70 \cdot 66$ & 57.56 & 1.38 \\
\hline & DFFB & $12 \cdot 77$ & $6 \cdot 75$ & 71.34 & 59.87 & 1.44 \\
\hline & DFFS & 21.64 & 10.67 & 59.35 & 49.96 & 1.44 \\
\hline & $\mathrm{CB}$ & $22 \cdot 78$ & $10 \cdot 98$ & $59 \cdot 16$ & 49.05 & 1.46 \\
\hline & CS & $27 \cdot 39$ & $13 \cdot 18$ & 53.83 & $42 \cdot 71$ & 1.25 \\
\hline \multirow[t]{6}{*}{ SH99250 (starch amylose content $=41.93 \%$ ) } & WM & $19 \cdot 19$ & $7 \cdot 70$ & 63.41 & 53.59 & $2 \cdot 11$ \\
\hline & SGF & $13 \cdot 32$ & $5 \cdot 18$ & 69.66 & 57.90 & 1.60 \\
\hline & DFFB & 14.72 & 7.04 & $71 \cdot 24$ & $60 \cdot 31$ & 1.30 \\
\hline & DFFS & 21.07 & 9.66 & 61.57 & $51 \cdot 35$ & 1.43 \\
\hline & CB & $22 \cdot 74$ & $10 \cdot 15$ & 59.72 & 48.98 & 1.89 \\
\hline & CS & $27 \cdot 86$ & $12 \cdot 30$ & 54.93 & 43.98 & $1 \cdot 19$ \\
\hline \multirow[t]{6}{*}{ CDC Candle (starch amylose content $=5.28 \%$ ) } & WM & $19 \cdot 12$ & $6 \cdot 64$ & $70 \cdot 92$ & $58 \cdot 69$ & 0.51 \\
\hline & SGF & $13 \cdot 29$ & $4 \cdot 38$ & 76.91 & 61.66 & 0.51 \\
\hline & DFFB & $15 \cdot 14$ & $6 \cdot 28$ & $79 \cdot 12$ & 64.39 & 0.43 \\
\hline & DFFS & 21.54 & 9.99 & 64.70 & 50.99 & 0.42 \\
\hline & CB & 22.97 & $9 \cdot 51$ & $67 \cdot 30$ & 54.34 & 0.54 \\
\hline & CS & $28 \cdot 32$ & 11.99 & $55 \cdot 81$ & $43 \cdot 53$ & 0.48 \\
\hline \multirow[t]{7}{*}{ CDC Fibar (starch amylose content $=0 \%$ ) } & WM & $18 \cdot 24$ & 9.66 & $62 \cdot 46$ & 52.06 & 1.03 \\
\hline & SGF & 14.06 & $5 \cdot 74$ & 68.07 & $51 \cdot 76$ & 0.47 \\
\hline & DFFB & $12 \cdot 05$ & $7 \cdot 86$ & 73.73 & 61.05 & 0.45 \\
\hline & DFFS & $23 \cdot 34$ & 11.90 & $60 \cdot 85$ & $46 \cdot 59$ & 0.76 \\
\hline & $\mathrm{CB}$ & $22 \cdot 38$ & $13 \cdot 41$ & $58 \cdot 46$ & $48 \cdot 68$ & 0.88 \\
\hline & CS & $29 \cdot 17$ & $15 \cdot 58$ & $53 \cdot 20$ & $42 \cdot 37$ & 0.90 \\
\hline & $\mathrm{P}$ & 43.51 & $2 \cdot 51$ & $12 \cdot 64$ & - & - \\
\hline
\end{tabular}

$\mathrm{db}$, Dry basis; WM, wholemeal; SGF, straight-grade flour; DFFB, dusted flour from bran; DFFS, dusted flour from shorts; CB, concentrated bran; CS, concentrated shorts; $P$, pearlings.

low-amylose cultivar CDC Fibar, as described by Harding et $a l .{ }^{(38)}$. These flour blends were used to prepare the scenarios indicated earlier: (1) WGF (low amylose) $v$. HA-DFF (high amylose); (2) SGF (low $\beta$-glucan) $v$. WGF (medium $\beta$-glucan) $v$. BF-BG (high $\beta$-glucan); (3) WGF (low insoluble fibre) $v$. BF-IDF (high insoluble fibre).

\section{Test meal processing, analysis and presentation}

Barley tortillas made only from barley flour and water, as developed previously at the Cereal Research Centre, Agriculture and Agri-Food Canada ${ }^{(45)}$, were chosen as the test food product, because they required no additional ingredients that could interfere with the results. The five selected flour blends were processed into tortillas in the Metabolic Kitchen Facility at the Richardson Centre for Functional Foods and Nutraceuticals (Winnipeg, Manitoba, Canada) using the following basic protocol: mix $200 \mathrm{~g}$ of flour with water for 5 min (GRL200 Mixer, Muzeen \& Blythe), rest dough at room temperature for $10 \mathrm{~min}$, sheet dough (National MFG Company), die cut to $14.5 \mathrm{~cm}$ diameter and cook on a $250^{\circ} \mathrm{C}$ tortilla grill (Bakery Equipment and Service Company). The water amount, sheeting thickness and cooking times were optimised for each flour blend to manage variation in the dough-handling properties between treatments. Tortilla moisture content was measured using the two-stage AACC International Approved

Table 2. Barley test meal treatment scenarios calculated to deliver varying amounts of fibre components in a single serving

\begin{tabular}{|c|c|c|c|c|c|c|}
\hline \multirow[b]{2}{*}{ Genotype } & \multirow[b]{2}{*}{ Milling fraction } & \multirow[b]{2}{*}{ Dry content per serving (g) } & \multicolumn{4}{|c|}{ Composition per serving (g) } \\
\hline & & & Total dietary fibre & IDF* $^{*}$ & $\beta$-Glucan & Available carbohydrate \\
\hline Fibar & SGF & 73.45 & $10 \cdot 33$ & $6 \cdot 11$ & 4.22 & 50 \\
\hline SH99250 & SGF, DFFS, DFFB & $72 \cdot 60$ & $10 \cdot 34$ & $6 \cdot 15$ & 4.19 & 50 \\
\hline Fibar & WM & $76 \cdot 85$ & $14 \cdot 32$ & $6 \cdot 82$ & 7.49 & 50 \\
\hline Fibar & CB, DFFB & 84.34 & $18 \cdot 41$ & 7.35 & 11.06 & 50 \\
\hline SH99250 & DFFS, DFFB & $76 \cdot 67$ & $14 \cdot 32$ & 7.67 & 6.65 & 50 \\
\hline Fibar & DFFB, PC & $82 \cdot 28$ & 19.43 & 7.99 & 11.43 & 50 \\
\hline Fibar & PC, DFFB & 88.37 & $24 \cdot 17$ & $10 \cdot 16$ & 14.01 & 50 \\
\hline Fibar & SGF, P & $92 \cdot 26$ & $19 \cdot 78$ & $15 \cdot 23$ & 4.55 & 50 \\
\hline Fibar & SGF, CS, P & $97 \cdot 86$ & 24.50 & 17.01 & 7.49 & 50 \\
\hline
\end{tabular}

IDF, insoluble dietary fibre; SGF, straight-grade flour; DFFS, dusted flour from shorts; DFFB, dusted flour from bran; WM, wholemeal; CB, concentrated bran; $\mathrm{P}$, pearlings; PC, Polycell Technologies high $\beta$-glucan fraction; CS, concentrated shorts.

* IDF was estimated for flour blend scenario calculations (IDF = total dietary fibre - $\beta$-glucan soluble fibre). All other components were calculated using analytical data obtained from the milling fractions. 
Table 3. Barley tortilla test meal treatment composition confirmed by analytical chemistry

\begin{tabular}{|c|c|c|c|c|c|c|c|c|}
\hline \multirow[b]{2}{*}{ Treatment } & \multirow[b]{2}{*}{$\begin{array}{c}\text { Dry content } \\
\text { per serving }(\mathrm{g})\end{array}$} & \multicolumn{7}{|c|}{ Composition per serving (g) } \\
\hline & & Total dietary fibre & IDF & $\beta$-Glucan & Available carbohydrate & Total starch & Resistant starch & Protein \\
\hline SGF & $75 \cdot 74$ & $10 \cdot 29$ & 7.55 & 4.50 & 50 & 38.34 & 0.45 & 13.67 \\
\hline WGF & $78 \cdot 24$ & $14 \cdot 28$ & $7 \cdot 43$ & 7.77 & 50 & 38.92 & 0.42 & $13 \cdot 28$ \\
\hline BF-BG & $83 \cdot 33$ & 18.03 & 7.47 & 11.55 & 50 & 38.47 & 0.85 & 14.71 \\
\hline BF-IDF & $108 \cdot 41$ & $26 \cdot 87$ & 19.64 & 8.56 & 50 & 39.92 & 0.68 & 21.50 \\
\hline HA-DFF & $76 \cdot 22$ & 14.14 & 8.29 & $6 \cdot 27$ & 50 & $40 \cdot 13$ & 1.41 & 12.08 \\
\hline
\end{tabular}

IDF, insoluble dietary fibre; SGF, straight-grade flour (low $\beta$-glucan/low IDF); WGF, wholegrain flour (medium $\beta$-glucan/low IDF); BF-BG, bran flour with high $\beta$-glucan/low IDF; BF-IDF, bran flour with high IDF/medium $\beta$-glucan; HA-DFF, high-amylose dusted flour fractions (medium $\beta$-glucan/low IDF).

Method $44-15 \mathrm{~A}^{(46)}$ and used to calculate the serving size required to deliver $50 \mathrm{~g}$ of available carbohydrate (therefore, serving sizes ranged from approximately seven to ten tortillas depending on the treatment; the average weight of each tortilla was $15.5 \mathrm{~g}$, and the average approximate diameter was $14.5 \mathrm{~cm}$ ). Full chemical analysis of the tortillas (using the methods described earlier for milling fraction analysis) confirmed the final composition of the prepared test meals (Table 3). A difference between the available carbohydrate and total starch content of the tortilla treatments was noted in the analysis. This difference was partly a result of the fact that glucose in starch is in an anhydrous form. When glucose is released after being exposed to amylases, it binds to another water molecule (per glucose), i.e. the molecular weight of glucose increases from 162 to $180 \mathrm{~g} / \mathrm{mol}$; therefore, the total starch expressed as available carbohydrate is $11 \cdot 1 \%$ higher. The remaining difference between available carbohydrate and starch content was likely due to free sugars in the tortilla samples, although these were not measured. Acid extraction of the tortilla samples was carried out $^{(47)}$, and viscosity measurements of the acid extracts were made using a Brookfield DV-II + Pro Viscometer (LV-4 spindle, $60 \mathrm{rpm}$; Brookfield Engineering Laboratories) to determine whether the tortilla samples differed in viscosity (see online supplementary Fig. S1). As can be seen in Fig. S1, the tortillas differed in viscosity; the high- $\beta$-glucan tortillas (BF-BG) were more viscous than the other treatments were.

Tortillas were stored frozen until use in the clinical trial. Before presenting test meals to the participants, tortillas were reheated in a Black \& Decker three-tier food steamer for 10 min wrapped in wax paper. Warmed tortillas were then wrapped in aluminium foil and placed in a Bravetti buffet server until served.

\section{Clinical trial}

A single-site, double-blind, randomised, controlled, cross-over study was conducted at the I. H. Asper Clinical Research Institute at St Boniface Hospital in Winnipeg, Manitoba, Canada.

There were a total of twelve participants, aged $19-35$ years with a glycated $\mathrm{Hb}$ of $<6 \%$ and BMI between 20 and $31 \mathrm{~kg} / \mathrm{m}^{2}$. They did not have any allergies to barley flour or any chronic diseases, such as CVD, hypertension, disorders affecting the GIT tract or thyroid disease, or require medications for these conditions. They did not require medication for glycaemic control or consume supplements which had an effect on blood glucose response. See Table 4 for details on the demographics of the participants.

The present study was performed according to the guidelines in the Declaration of Helsinki. Ethics approval for the clinical trial was obtained from the University of Manitoba Biomedical Research Ethics Board and the St Boniface Hospital Research Review Committee. The present study was registered on http://www.clinicaltrials.gov (NCT00831285). The first participant's initial visit was on 6 May 2009, and the final visit of the last participant was completed on 18 February 2010. Participants provided written informed consent before undergoing any study-related procedures. A total of thirteen participants provided written informed consent, but one participant dropped out of the study after the first visit due to a physiological aversion to blood draws. The remaining twelve participants came in for six visits that were separated by at least 1 week. The test products consumed included one of five different barley tortillas (Table 3) or an orangeflavoured Trutol Glucose Tolerance Beverage containing $50 \mathrm{~g}$ dextrose (from Fisher Scientific) assigned in random order. Participants arrived at each clinic visit in the morning after an overnight fast. Blood was collected at fasting and at 15 , 30, 45, 60, 120 and $180 \mathrm{~min}$ after the first bite/sip of the test product. Blood was collected into lithium heparin tubes for

Table 4. Demographics

\begin{tabular}{|c|c|c|c|c|}
\hline & $n$ & Average & Minimum & Maximum \\
\hline Male & 7 & & & \\
\hline Female & 5 & & & \\
\hline \multicolumn{5}{|l|}{ Medications/supplements } \\
\hline None & 7 & & & \\
\hline $\begin{array}{l}\text { Acetylsalicylic acid, } \\
81 \text { mg daily }\end{array}$ & 1 & & & \\
\hline Tylenol plain & 1 & & & \\
\hline Valcyclovir, 1000 mg & 1 & & & \\
\hline $\begin{array}{l}\text { Ventolin, two puffs as } \\
\text { required }\end{array}$ & 1 & & & \\
\hline $\begin{array}{l}\text { Vitamin } \mathrm{B}_{12}, \mathrm{Fe} \text { and } \\
\text { Centrum multivitamin, } \\
\text { daily }\end{array}$ & 1 & & & \\
\hline Age (years) & & 27 & 19 & 35 \\
\hline BMI $\left(\mathrm{kg} / \mathrm{m}^{2}\right)$ & & $23 \cdot 8$ & 20 & 31 \\
\hline Waist circumference $(\mathrm{cm})$ & & $80 \cdot 6$ & $66 \cdot 9$ & $107 \cdot 0$ \\
\hline
\end{tabular}




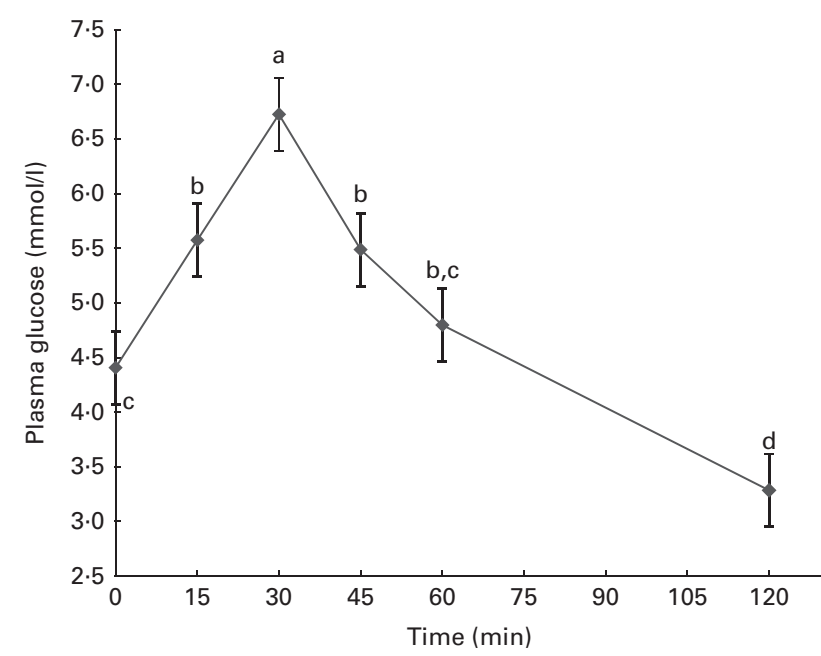

Fig. 1. Plasma glucose concentrations after consuming glucose reference drink. Values are means ( $n 12)$, with their standard errors represented by vertical bars. ${ }^{a, b, c, d}$ Mean values with unlike letters were significantly different $(P<0.05)$.

glucose analysis and EDTA tubes for hormone analysis. Dipetidyl peptidase IV inhibitor (Millipore) and Halt protease inhibitor (Pierce) were added immediately to the EDTA tubes and centrifuged at $1800 \mathrm{~g}$ for $10 \mathrm{~min}$ at $4^{\circ} \mathrm{C}$ to separate plasma. Plasma was stored at $-80^{\circ} \mathrm{C}$ until analysis.

Plasma glucose concentrations at each time point were determined using an enzymatic colorimetric kit (Genzyme Diagnostics). Plasma insulin concentrations at each time point were measured using an ultrasensitive ELISA kit from Alpco Diagnostics. Active GLP-1 and PYY concentrations at the $0,30,60,120$ and 180 time points were determined using an electrochemiluminescent immunoassay from Meso Scale Discovery. All samples and respective standards were analysed in triplicate for glucose and in duplicate for the hormones with a percentage $\mathrm{CV}$ of less than $10 \%$. Incremental AUC (iAUC) for glucose, insulin, GLP-1 and PYY was calculated as described by Brouns et al. ${ }^{(48)}$. GI was calculated by dividing the glucose iAUC for each tortilla by the glucose drink iAUC and multiplying by 100 .

\section{Randomisation procedures}

A list of unique product code numbers was prepared to ensure that volunteers received the treatments in random order. Randomisation was performed by the research team. After randomisation occurred, the study nurse was responsible for ensuring that the assigned randomisation number was applied to all study documentation for that particular volunteer and for dispensing the foods with the matching randomisation number.

\section{Blinding}

Although participants knew whether they were consuming a food product or a liquid, both the research team and the volunteer were blinded from the time of randomisation and for the duration of the study in regards to which tortilla they ate during any given visit.

\section{Statistical analysis}

Mäkeläinen et $a l{ }^{(49)}$ incorporated similar methodology to explore of the effect of $\beta$-glucan on the glycaemic and insulin index with oat products. Their findings showed that $n 10$ participants were sufficient for attaining evaluable results. We recruited $n 12$ to allow us to reach our target sample while accounting for a small percentage of withdrawals by subjects who might be unable to complete the study.

The present study was designed to examine three effects: (1) the effect of low $v$. high amylose; (2) the effect of low, medium and high $\beta$-glucan; and (3) the effect of low $v$. high IDF on glycaemic response. For effects (1) and (3), paired $t$ tests were used to determine differences in GI, iAUC and percentage change from baseline at $30 \mathrm{~min}$ between the groups. For effect (3), data were analysed using PROC MIXED with tortilla as a fixed effect and subject as a random variable using the SAS system version 9.2 (SAS Institute Inc.). PROC MIXED repeated measures analysis was used to determine differences between time points. Differences among means were determined by Lsmeans.

The primary outcome was iAUC for glucose. The secondary outcome was iAUC for insulin. Additional outcomes were GI and percentage change from baseline at $30 \mathrm{~min}$.

\section{Results}

\section{Glycaemic index}

Foods can be classified as low GI $(<55)$, medium GI (56-69) or high GI $(>70)$. The plasma glucose curve after consumption of the glucose reference drink is shown in Fig. 1. The WGF tortillas (low amylose, medium $\beta$-glucan and low IDF) were classified as medium GI, whereas all of the other tortilla treatments were low GI (Table 5 ). The high- $\beta$-glucan (BF-BG) tortilla treatment had a 56-60\% lower GI as compared to both the low- $\beta$-glucan (SGF) and medium- $\beta$-glucan (WGF) tortilla treatments (Table 5). There was no significant

Table 5. Glycaemic index (GI) of barley tortillas

(Mean values with their standard errors, $n$ 12)

\begin{tabular}{llr}
\hline & \multicolumn{2}{c}{ Gl values } \\
\cline { 2 - 3 } Treatment & Mean & SEM \\
\hline WGF & 57.3 & 13.5 \\
HA-DFF & 39.2 & 14.2 \\
SGF & $51.8^{\mathrm{a}}$ & 11.8 \\
WGF & $57.3^{\mathrm{a}}$ & 11.4 \\
BF-BG & $22.7^{\mathrm{b}}$ & 11.4 \\
WGF & 57.3 & 13.5 \\
BF-IDF & 40.9 & 11.0 \\
\hline
\end{tabular}

WGF, wholegrain flour (low amylose/medium $\beta$-glucan/low insoluble dietary fibre); HA-DFF, high-amylose dusted flour fractions (high amylose); SGF, straightgrade flour (low $\beta$-glucan); BF-BG, bran flour with high $\beta$-glucan (high $\beta$-glucan); BF-IDF, bran flour with high insoluble dietary fibre (high insoluble dietary fibre).

${ }^{\mathrm{a}, \mathrm{b}}$ Mean values with unlike superscript letters were significantly different $(P<0.05)$. 
difference in GI between the low-amylose (WGF) and high-amylose (HA-DFF) treatments (Table 5) or between the low-IDF (WGF) and high-IDF (BF-IDF) treatments (Table 5).

\section{Plasma glucose}

There was no difference in the fasting plasma glucose concentrations between the participants at each visit (Fig. 2(A), (D) and $(G)$ ). There was no significant increase in plasma glucose from baseline at any time point after eating the high- $\beta$-glucan tortillas (Fig. 2(D)). Plasma glucose concentrations were $9-16 \%$ higher at $30 \mathrm{~min}$ and $7-15 \%$ higher at $45 \mathrm{~min}$ as compared to baseline levels for all of the other treatments, but plasma glucose concentrations returned to baseline levels by $45 \mathrm{~min}$ for the high-IDF tortillas (Fig. 2(A), (D) and (G)).

The high- $\beta$-glucan treatment had a $61 \%$ lower glucose iAUC as compared to the low- $\beta$-glucan treatment (Fig. 2(E)).
The high- $\beta$-glucan treatment had a percentage change from baseline at $30 \mathrm{~min}$ that was 3.9-5.1 times lower than both the low- and medium- $\beta$-glucan treatments (Fig. 2(F)). There was no difference in the glucose iAUC or percentage change from baseline at $30 \mathrm{~min}$ between the low- and high-amylose treatments (Fig. 2(B) and (C)) or between the low- and high-IDF treatments (Fig. 2(H) and (I)).

\section{Plasma insulin}

Fasting plasma insulin concentrations were not significantly different between visits (Fig. 3(A), (D) and (G)). All treatments had significantly higher insulin concentrations at $15-60 \mathrm{~min}$ as compared to baseline levels (Fig. 3(A), (D) and (G)). The high-amylose and high- $\beta$-glucan treatments returned to baseline levels by $120 \mathrm{~min}$, whereas the low- $\beta$-glucan treatment returned to baseline by $180 \mathrm{~min}$. Plasma insulin concentrations
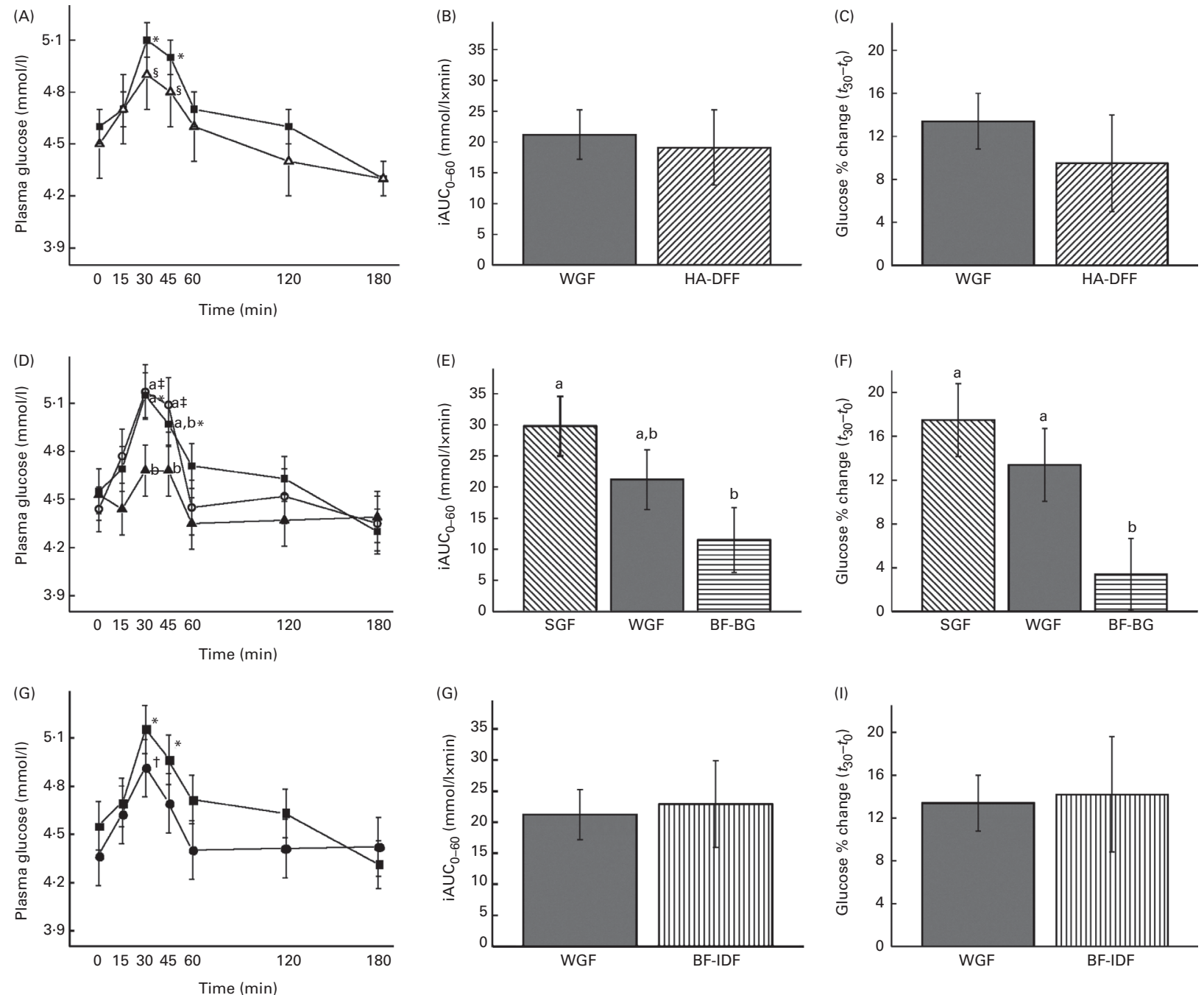

Fig. 2. Effect of barley tortillas on plasma glucose concentrations. Low $v$. high amylose $(A-C)$; low $v$. medium $v$. high $\beta$-glucan (D-F); low $v$. high insoluble dietary fibre (IDF) $(G-I)$. Plasma glucose over time (A, D, G); glucose incremental AUC (iAUC) $(B, E, H)$; percentage change in plasma glucose from baseline at 30 min $(C, F, I)$. WGF (--), wholegrain flour (low amylose/medium $\beta$-glucan/low IDF); HA-DFF ( --$)$, high-amylose dusted flour fractions (high amylose); SGF (-O-), straight-grade flour (low $\beta$-glucan); BF-BG $(--)$, bran flour with high $\beta$-glucan (high $\beta$-glucan); BF-IDF ( - -), bran flour with high IDF. Values are means ( $n$ 12), with their standard errors represented by vertical bars. ${ }^{a, b}$ Mean values with unlike letters were significantly different $(P<0 \cdot 05)$. ${ }^{\star}$ Mean value was significantly different from baseline for WGF treatment $(P<0.05)$. $†$ Mean value was significantly different from baseline for BF-IDF treatment $(P<0.05)$. $\ddagger$ Mean value was significantly different from baseline for SGF treatment $(P<0.05)$. $§$ Mean value was significantly different from baseline for HA-DFF treatment $(P<0.05)$. 

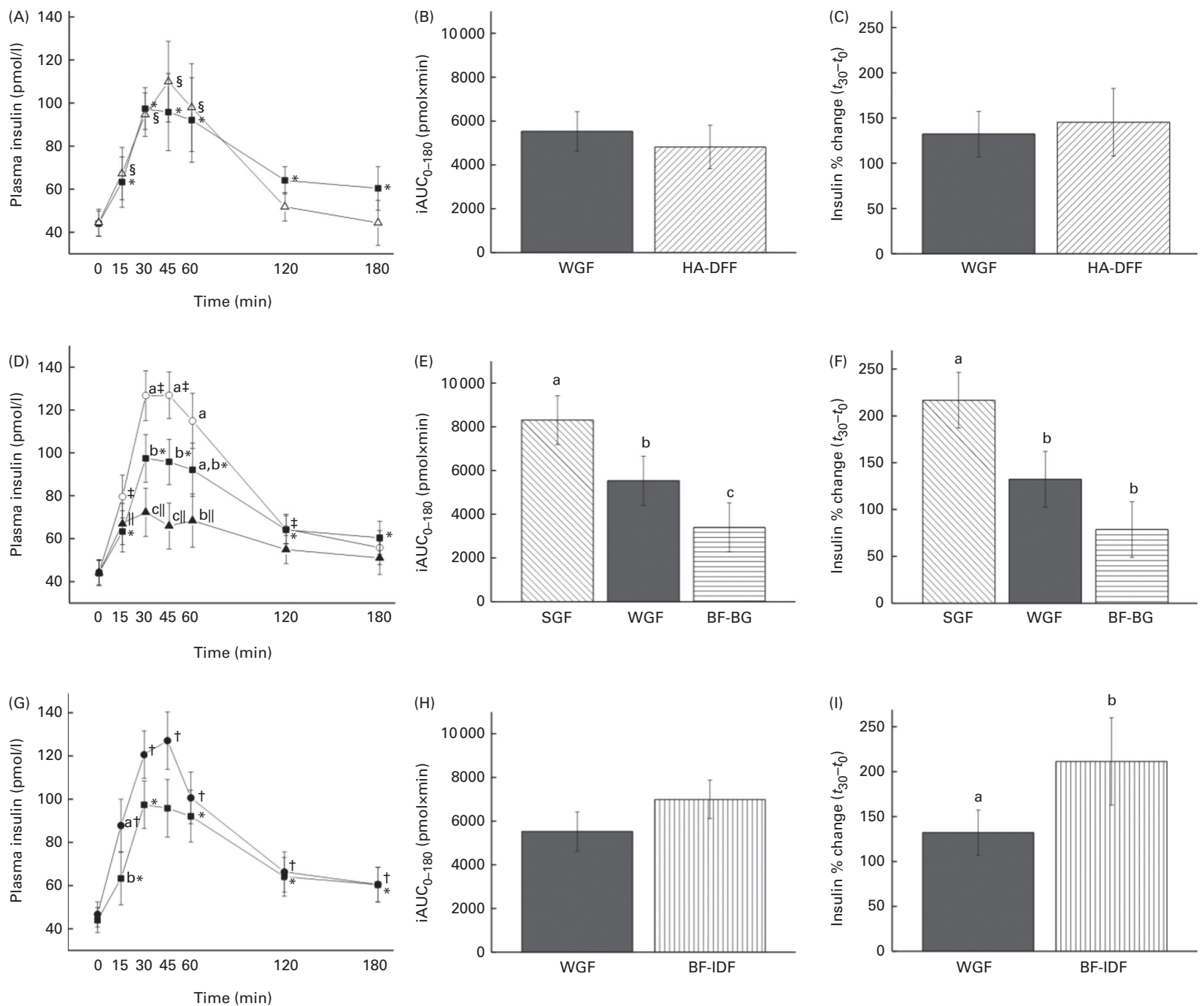

Fig. 3. Effect of barley tortillas on plasma insulin concentrations. Low $v$. high amylose (A-C); low $v$. medium $v$. high $\beta$-glucan (D-F); low $v$. high insoluble dietary fibre (IDF) (G-I). Plasma insulin overtime (A, D, G); insulin incremental (iAUC) (B, E, H); percentage change in plasma insulin from baseline at 30 min (C, F, I). WGF (-)), wholegrain flour (low amylose/medium $\beta$-glucan/low IDF); HA-DFF (- - ), high-amylose dusted flour fractions (high amylose); SGF (-o-), straight-grade flour (low $\beta$-glucan); BF-BG ( $\_$), bran flour with high $\beta$-glucan (high $\beta$-glucan); BF-IDF (-๑), bran flour with high IDF. Values are means ( $n$ 12), with their standard errors represented by vertical bars. ${ }^{\mathrm{a}, \mathrm{b}, \mathrm{c}}$ Mean values with unlike letters were significantly different $(P<0.05)$. ${ }^{*}$ Mean value was significantly different from baseline for WGF treatment $(P<0.05)$. † Mean value was significantly different from baseline for BF-IDF treatment $(P<0.05)$. $\ddagger$ Mean value was significantly different from baseline for SGF treatment $(P<0.05)$. § Mean value was significantly different from baseline for HA-DFF treatment $(P<0.05)$. $\|$ Mean value was significantly different from baseline for BF-BG treatment $(P<0.05)$.

were still higher than baseline levels at 180 min after eating the low-amylose/medium- $\beta$-glucan treatments with low IDF (WGF) and high IDF (BF-IDF).

The high- $\beta$-glucan tortilla treatment had a $39 \%$ lower iAUC for insulin as compared to the medium- $\beta$-glucan tortillas, which in turn had a $33 \%$ lower insulin iAUC as compared to the low- $\beta$-glucan treatment (Fig. 3(E)). The low- $\beta$-glucan tortillas had a $64-176 \%$ higher percentage change from baseline at $30 \mathrm{~min}$ for plasma insulin as compared to both the mediumand high- $\beta$-glucan tortillas (Fig. 3(F)). There was no significant difference between the high- and low-IDF tortillas in terms of insulin iAUC (Fig. 3(H)), but the high-IDF tortillas had a higher percentage change of plasma insulin from baseline at $30 \mathrm{~min}$ as compared to the low-IDF tortillas (Fig. 3(I)). There was no significant difference in insulin iAUC or percentage change from baseline at $30 \mathrm{~min}$ between the low- and highamylose treatments (Fig. 3(B) and (C)).

\section{Plasma glucagon-like peptide-1}

There was no significant difference in fasting GLP-1 concentrations between the treatments (Fig. 4(A), (D) and (G)). All of the treatments had significantly higher plasma GLP-1 concentrations at $30 \mathrm{~min}$. The GLP-1 concentrations after eating the high-amylose (HA-DFF) and high- $\beta$-glucan (BF-BG) tortillas returned to baseline by $60 \mathrm{~min}$. The low-amylose/medium$\beta$-glucan/low-IDF (WGF) and low- $\beta$-glucan (SGF) tortillas had plasma GLP-1 concentrations that were not significantly different from baseline by $120 \mathrm{~min}$, but the GLP-1 concentrations after eating the low- $\beta$-glucan tortillas were higher than 
(A)

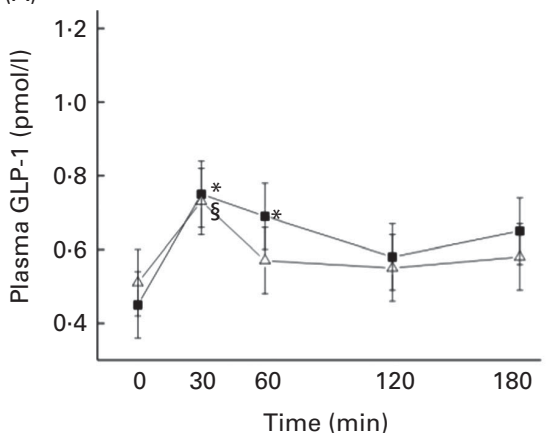

(D)

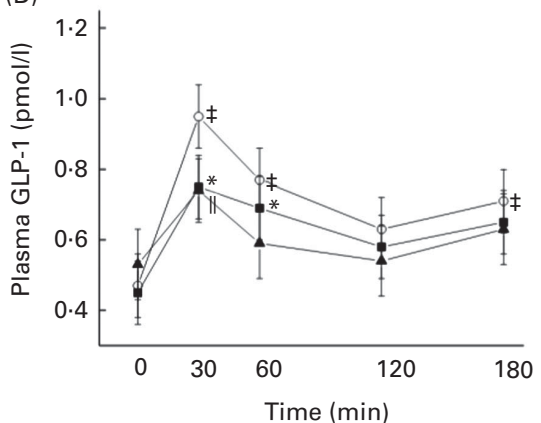

(G)

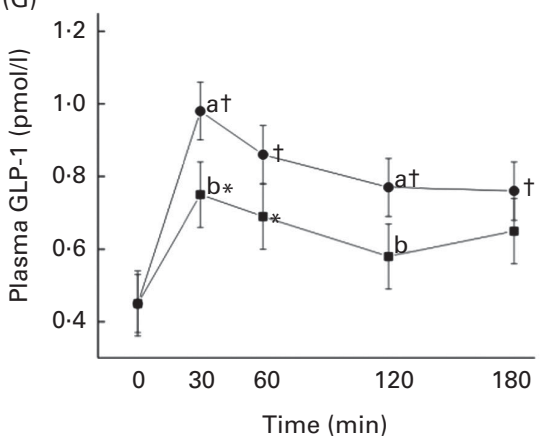

(B)

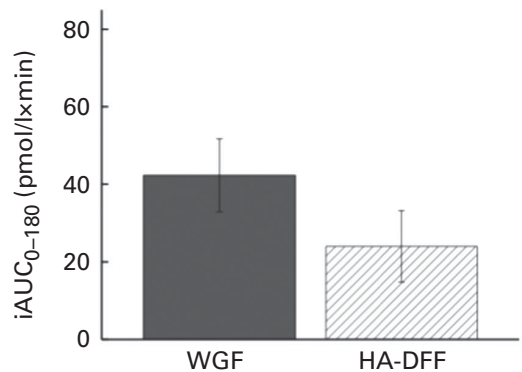

(E)

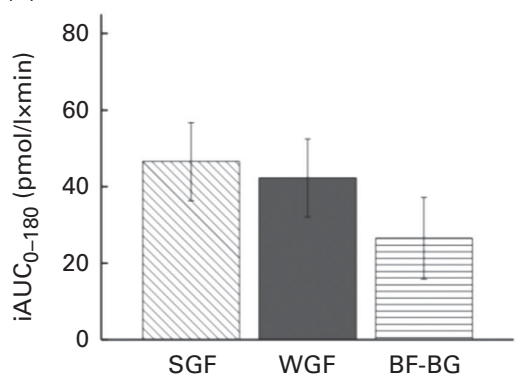

(H)

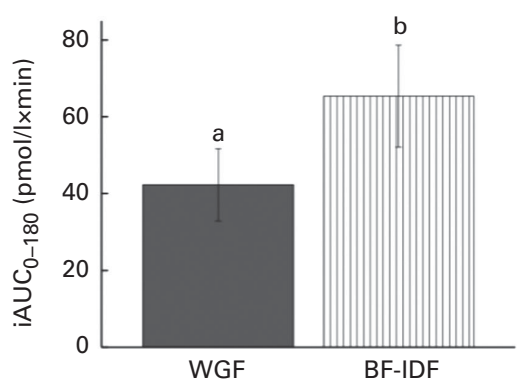

(C)

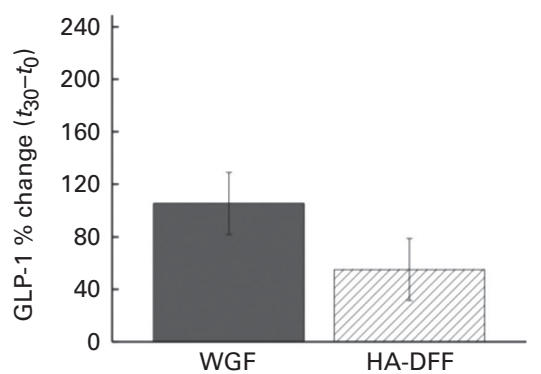

(F)

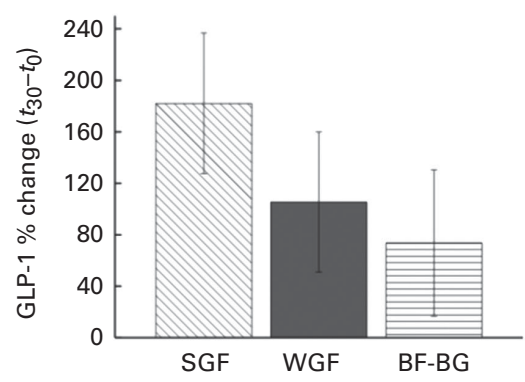

(I)

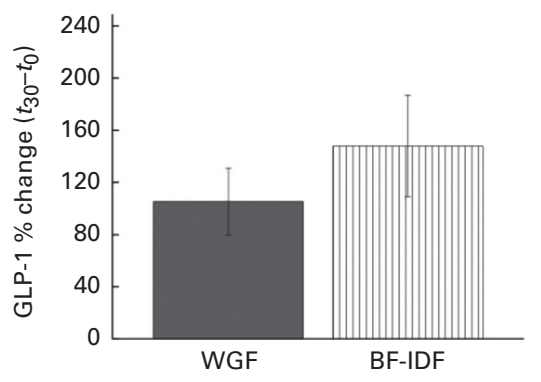

Fig. 4. Effect of barley tortillas on plasma glucagon-like peptide-1 (GLP-1) concentrations. Low $v$. high amylose (A-C); low $v$. medium $v$. high $\beta$-glucan (D-F); low v. high insoluble dietary fibre (G-I). Plasma GLP-1 over time (A, D, G); GLP-1 incremental AUC (iAUC) (B, E, H); percentage change in plasma GLP-1 from baseline at $30 \mathrm{~min}(\mathrm{C}, \mathrm{F}, \mathrm{I})$. WGF (-E), wholegrain flour (low amylose/medium $\beta$-glucan/low IDF); HA-DFF ( $\triangle$ ), high-amylose dusted flour fractions (high amylose); SGF (-O), straight-grade flour (low $\beta$-glucan); BF-BG $(-\leftarrow)$, bran flour with high $\beta$-glucan (high $\beta$-glucan); BF-IDF (--), bran flour with high IDF. Values are means ( $n$ 12), with their standard errors represented by vertical bars. ${ }^{a, b}$ Mean values with unlike letters were significantly different $(P<0.05)$. ${ }^{*}$ Mean value was significantly different from baseline for WGF treatment $(P<0.05)$. † Mean value was significantly different from baseline for BF-IDF treatment $(P<0.05)$. $\ddagger$ Mean value was significantly different from baseline for SGF treatment $(P<0.05)$. $§$ Mean value was significantly different from baseline for HA-DFF treatment $(P<0.05)$. $\|$ Mean value was significantly different from baseline for BF-BG treatment $(P<0.05)$.

baseline at $180 \mathrm{~min}$. The high-IDF tortillas had higher GLP-1 concentrations than baseline at every time point (Fig. 4(G)).

There was no significant difference in GLP-1 iAUC or percentage change from baseline at $30 \mathrm{~min}$ between the low- and high-amylose treatments (Fig. 4(B) and (C)) or between the low-, medium- and high- $\beta$-glucan treatments (Fig. 4(E) and (F)). The high-IDF treatment had a 54\% higher GLP-1 iAUC as compared to the low-IDF treatment (Fig. 4(H)). There was no difference between the low- and high-IDF treatments in the percentage change from baseline at $30 \mathrm{~min}$ (Fig. 4(I)).

\section{Plasma peptide $Y Y$}

There was no significant difference in plasma PYY concentrations between time points or between treatments. There was also no significant difference in PYY iAUC or percentage change from baseline at $30 \mathrm{~min}$ between treatments (data not shown).

\section{Discussion}

Overall, the results of the present research provide knowledge about the effects of high-fibre barley consumption on one of the physiological factors that is associated with heart disease and diabetes: postprandial glucose and insulin response. The Canadian Diabetes Association recommends that people with diabetes replace high-GI foods with low-GI foods to improve glycaemic control ${ }^{(1)}$, and the present study showed that barley tortillas made with high $\beta$-glucan or high IDF are low-GI foods. All of the barley tortillas elicited lower PPGR as compared to the glucose drink; however, increasing the 
amount of barley $\beta$-glucan in the tortillas improved PPGR further, whereas increasing the amylose or insoluble fibre content had no effect on PPGR.

Using barley flour from a genotype that is rich in amylose (SH99250, which has $42 \%$ amylose content in its extracted starch) did not improve PPGR, which is in line with the report by Granfeldt et al. ${ }^{(15)}$. High-amylose rice (23\% amylose content) ${ }^{(50)}$, high-amylose maize starch $(70-75 \%$ amylose content $)^{(51)}$ and high-amylose wheat $(38 \%$ amylose content) ${ }^{(52)}$ have been shown to reduce PPGR, but these foods contain negligible amounts of $\beta$-glucan. Any benefit of increasing amylose content may be masked by the effects of $\beta$-glucan in foods that contain barley. Moreover, the tortillas made with high-amylose barley flour contained $1.41 \mathrm{~g}$ resistant starch per serving as compared to $0.42 \mathrm{~g}$ in the low-amylose tortillas. Therefore, the dosage of resistant starch may not have been sufficient to reduce the glycaemic response in general and/or to reduce the glycaemic response further than what was achieved by the $\beta$-glucan content. The present results do not support the use of barley varieties based on the amylose:amylopectin ratio to improve the PPGR of food products made from barley flour.

The European Food Safety Authority has approved a health claim for $\beta$-glucans from oats or barley for their beneficial effect of improving PPGR ${ }^{(10)}$. According to this claim, a beneficial physiological effect requires the consumption of $4 \mathrm{~g}$ of $\beta$-glucan from oat or barley for each $30 \mathrm{~g}$ of available carbohydrate consumed per meal. A recent review ${ }^{(53)}$ demonstrated that in order to achieve a significant lowering of PPGR, $3 \mathrm{~g}$ of $\beta$-glucan per meal is sufficient if the $\beta$-glucan is consumed as intact cooked or fermented grains, and $4 \mathrm{~g}$ of $\beta$-glucan per meal is sufficient if the $\beta$-glucan is processed. The present tortilla study used $\beta$-glucan doses of $4.5,7.8$ and $11.6 \mathrm{~g}$ per $50 \mathrm{~g}$ of available carbohydrate. Although all of the barley tortilla treatments showed a $20 \%$ reduction in glucose response as compared to consuming glucose alone, the maximum reduction was observed with the treatments that contained the highest amount of $\beta$-glucan (which also corresponded with the highest viscosity). These results suggested that $\beta$-glucan doses of more than $4 \mathrm{~g}$ could be more effective at blunting glycaemic response, and this warrants future clinical study to examine the dose-dependent effect of $\beta$-glucan on PPGR. Considering the acute nature of the present study, the role of $\beta$-glucan fermentation products, such as acetate, propionate and butyrate, on glucose metabolism could not be determined $^{(54,55)}$. Accordingly, slowed glucose absorption as a result of increased gut viscosity might have been responsible for the acute effects that were observed ${ }^{(56)}$; this idea was supported by the supplementary viscosity data. Future long-term studies in people with type 2 diabetes are needed to validate these effects and to determine whether there is an added benefit of increased-fermentation products in the lower GIT for maintaining lower blood glucose in the longer term.

Although previous studies have shown that the consumption of IDF is associated with a reduced risk of type 2 diabetes ${ }^{(57)}$ and lowered PPGR 75 min after consuming a meal ${ }^{(58)}$, in the present study, the differential intake of low $v$. high IDF ( $7 \cdot 4 v$. 19.6\% flour basis, respectively) did not alter glycaemic or insulinemic responses. As in a previous report ${ }^{(59)}$, the present study showed that insulin concentrations remained elevated during the study period, whereas glucose concentrations returned to baseline. We speculate that this discrepancy might have resulted from the fact that low levels of insulin may have been required to control the slow release of glucose. In addition, in the present study, the high-IDF tortillas elicited the highest GLP-1 response, which suggested increased satiety. Future studies should include visual analogue scales and measure ad libitum energy intake at the next meal(s) to determine if these highIDF tortillas result in increased satiety and/or reduced energy intake.

Overall, the present results indicated that the type of dietary fibre present in barley flour and its milling fractions could alter postprandial glucose and insulin responses. The selection of high- $\beta$-glucan barley genotypes and specialised milling fractions could be used to optimise barley-based functional foods to reduce PPGR. This opens new opportunities and novel ways to optimise the health benefits of barley tortillas as low-GI foods. The results of the present acute study indicated that barley food products enriched with $\beta$-glucan could be useful as part of a dietary approach for controlling and improving PPGR in humans. The results also indicated that barley tortillas with high IDF could possibly improve satiety, which has implications for weight control and obesity management. The data also supported the hypothesis that increasing $\beta$-glucan intake specifically is beneficial for helping control PPGR. More studies using a wholefoods approach for glycaemic control are needed for barley foods and wholegrain foods in general.

\section{Supplementary material}

To view supplementary material for the present article, please visit http://dx.doi.org/10.1017/S0007114515000367

\section{Acknowledgements}

The authors would like to thank and acknowledge Dr Brian Rossnagel, University of Saskatchewan, for providing the barley samples for the study and the AAFC staff, particularly Tracy Exley and Camille Rhymer, for their technical support.

The authors would also like to acknowledge Angela Wilson (clinical trial coordinator/nurse), Brenda Wright and Danielle Stringer for their technical support and Alanna Baldwin for preparing the initial regulatory documents for approval by the University of Manitoba Research Ethics Board and St Boniface General Hospital Research Review Committee.

Funding for the present project was provided by Agriculture and Agri-Food Canada as well as the Alberta Barley Commission (MII 53755). Additionally, H. B. received a postdoctoral fellowship from the Manitoba Health Research Council.

The authors' contributions are as follows: N. A. was the principal investigator of the study, conceived and designed the study, conducted the food development and analysis, created the treatments, designed the clinical trial and prepared 
the manuscript; H. B. prepared the amendments, sought the necessary regulatory approvals for study-related documents, recruited participants, screened participants, administered test products to participants, performed pre-analytical blood preparation, analysed blood samples, performed the statistical analysis of the clinical trial data and drafted the sections of the manuscript that describe the clinical trial; J. S. contributed to the data analysis and the manuscript preparation; S. J. T. contributed to the manuscript preparation and editing; P. Z . provided oversight for the clinical trial and sample analyses and edited the manuscript; and C. T. contributed to the design of the clinical trial, provided oversight for the clinical trial and edited the manuscript.

There are no conflicts of interest.

\section{References}

1. Dworatzek P, Arcudi K, Gougeon R, et al. (2013) Canadian Diabetes Association clinical practice guidelines for the prevention and management of diabetes in Canada: nutrition therapy. Can J Diabetes 37, S45-S55.

2. Ames N, Rhymer C, Rossnagel B, et al. (2006) Utilization of diverse hulless barley properties to maximize food product quality. Cereal Foods World 51, 23-28.

3. Jacobs M, Izydorczyk M, Preston K, et al. (2008) Evaluation of baking procedures for incorporation of barley roller milling fractions containing high levels of dietary fibre into bread. J Sci Food Agric 88, 558-568.

4. Gill S, Vasanthan T, Ooraikul B, et al. (2002) Wheat bread quality as influenced by the substitution of waxy and regular barley flours in their native and extruded forms. J Cereal Sci 36, 219-237.

5. Chaudhary V \& Weber F (1990) Barley bran flour evaluated as dietary fiber ingredient in wheat bread. Cereal Foods World 35, 560-562.

6. Kaur G, Sharma S, Nagi HP, et al. (2012) Functional properties of pasta enriched with variable cereal brans. J Food Sci Technol 49, 467-474.

7. Sudha M, Vetrimani R \& Leelavathi K (2007) Influence of fibre from different cereals on the rheological characteristics of wheat flour dough and on biscuit quality. Food Chem 100, $1365-1370$.

8. Lagasse S, Hatcher D, Dexter J, et al. (2006) Quality characteristics of fresh and dried white salted noodles enriched with flour from hull-less barley genotypes of diverse amylose content. Cereal Chem 83, 202-210.

9. Prakash J, Naik HR, Hussain SZ, et al. (2015) Effect of processing conditions on the quality characteristics of barley chips. J Food Sci Technol 52, 294-302.

10. European Food Safety Authority (2011) Scientific opinion on the substantiation of health claims related to beta-glucans from oats and barley and maintenance of normal blood LDL-cholesterol concentrations (ID 1236, 1299), increase in satiety leading to a reduction in energy intake (ID 851, 852 ), reduction of post-prandial glycaemic responses (ID 821-824), and "digestive function" (ID 850) pursuant to Article 13(1) of Regulation (EC) no. 1924/2006. EFSA J 9, 2207-2228.

11. Byrnes SE, Miller JC \& Denyer GS (1995) Amylopectin starch promotes the development of insulin resistance in rats. J Nutr 125, 1430-1437.

12. Behall KM, Scholfield DJ \& Canary J (1988) Effect of starch structure on glucose and insulin responses in adults. $A m \mathrm{~J}$ Clin Nutr 47, 428-432.
13. Goddard MS, Young G \& Marcus R (1984) The effect of amylose content on insulin and glucose responses to ingested rice. Am J Clin Nutr 39, 388-392.

14. Hospers J, Van Amelsvoort J \& Westrate J (1994) Amylose-toamylopectin ratio in pastas affects postprandial glucose and insulin responses and satiety in males. J Food Sci 59, $1144-1149$.

15. Granfeldt Y, Liljeberg H, Drews A, et al. (1994) Glucose and insulin responses to barley products: influence of food structure and amylose-amylopectin ratio. Am J Clin Nutr 59, 1075-1082.

16. Bourdon I, Yokoyama W, Davis P, et al. (1999) Postprandial lipid, glucose, insulin, and cholecystokinin responses in men fed barley pasta enriched with beta-glucan. Am J Clin Nutr 69, 55-63.

17. Kim H, Behall K, Vinyard B, et al. (2006) Short-term satiety and glycemic response after consumption of whole grains with various amounts of beta-glucan. Cereal Foods World 51, 29-33.

18. Liljeberg HG, Granfeldt YE \& Bjorck IM (1996) Products based on a high fiber barley genotype, but not on common barley or oats, lower postprandial glucose and insulin responses in healthy humans. J Nutr 126, 458-466.

19. Tosh SM (2014) Effects of oats on carbohydrate metabolism. In Oats Nutrition and Technology, pp. 281-297. Oxford, UK: Wiley Blackwell.

20. Marciani L, Gowland PA, Spiller RC, et al. (2001) Effect of meal viscosity and nutrients on satiety, intragastric dilution, and emptying assessed by MRI. Am J Physiol Gastrointest Liver Physiol 280, G1227-G1233.

21. Braaten JT, Wood PJ, Scott FW, et al. (1991) Oat gum lowers glucose and insulin after an oral glucose load. Am J Clin Nutr 53, $1425-1430$

22. Hlebowicz J, Wickenberg J, Fahlstrom R, et al. (2007) Effect of commercial breakfast fibre cereals compared with corn flakes on postprandial blood glucose, gastric emptying and satiety in healthy subjects: a randomized blinded crossover trial. Nutr J 6, 22.

23. Panahi S, Ezatagha A, Temelli F, et al. (2007) Beta-glucan from two sources of oat concentrates affect postprandial glycemia in relation to the level of viscosity. J Am Coll Nutr 26, 639-644.

24. Izydorczyk M \& Dexter J (2008) Barley beta-glucans and arabinoxylans: molecular structure, physicochemical properties, and uses in food products - a review. Food Res Int 41, $850-868$.

25. Andersson A, Lampi A, Nystrom L, et al. (2008) Phytochemical and dietary fiber components in barley varieties in the HEALTHGRAIN Diversity Screen. J Agric Food Chem 56, 9767-9776.

26. Andersson A, Andersson R, Autio K, et al. (1999) Chemical composition and microstructure of two naked waxy barleys. J Cereal Sci 30, 183-191.

27. Andersson A, Elfverson C, Andersson R, et al. (1999) Chemical and physical characteristics of different barley samples. J Sci Food Agric 79, 979-986.

28. Oscarsson M, Andersson R, Salomonsson A, et al. (1996) Chemical composition of barley samples focusing on dietary fibre components. J Cereal Sci 24, 161-170.

29. Aman P \& Newman C (1986) Chemical composition of some different types of barley grown in Montana, U.S.A. J Cereal Sci 4, 133-141.

30. Yalcin E, Celik S, Akar T, et al. (2007) Effects of genotype and environment on beta-glucan and dietary fiber contents of hull-less barleys grown in Turkey. Food Chem 101, $171-176$. 
31. Panfili G, Fratianni A, Di Criscio T, et al. (2008) Tocol and beta-glucan levels in barley varieties and in pearling byproducts. Food Chem 107, 84-91.

32. Bhatty R (1999) Beta-glucan and flour yield of hull-less barley. Cereal Chem 76, 314-315.

33. Zheng G, Rossnagel B, Tyler R, et al. (2000) Distribution of beta-glucan in the grain of hull-less barley. Cereal Chem 77, 140-144.

34. Klamczynski A \& Czuchajowska Z (1999) Quality of flours from waxy and nonwaxy barley for production of baked products. Cereal Chem 76, 530-535.

35. Izydorczyk M, McMillan T, Kletke J, et al. (2011) Effects of pearling, grinding conditions, and roller mill flow on the yield and composition of milled products from hull-less barley. Cereal Chem 88, 375-384.

36. Izydorczyk M, Dexter J, Desjardins R, et al. (2003) Roller milling of Canadian hull-less barley: optimization of roller milling conditions and composition of mill streams. Cereal Chem 80, 637-644.

37. Schoch TJ (1964) Iodimetric determination of amylose. Potentiometric titration: standard method. In Methods of Carbohydrate Chemistry, pp. 157-160 [RL Whistler, editor]. Orlando, FL: Academic Press.

38. Harding S, Storsley J, Thandapilly SJ, et al. (2013) Lower 30 minute serum insulin in healthy Sprague-Dawley rats consuming chips from specific barley flour blends. Cereal Chem 90, 474-479.

39. AACC International (1999) Method 32-07.01. Soluble, insoluble, and total dietary fiber in food and food products. In Approved Methods of Analysis. St Paul, MN: AACC International.

40. AACC International (1999) Method 32-23.01. Beta-glucan content of barley and oats-rapid enzymatic procedure. In Approved Methods of Analysis. St Paul, MN: AACC International.

41. AACC International (1999) Method 76-13.01. Total starch assay procedure (megazyme amyloglucosidase/ alpha-amylase method). In Approved Methods of Analysis. St Paul, MN: AACC International.

42. AACC International (editor) (2002) Method 32-40.01. Resistant Starch in Starch Samples and Plant Materials, 11th ed. St Paul, MN: AACC International.

43. McCleary BV (2007) An integrated procedure for the measurement of total dietary fibre (including resistant starch), non-digestible oligosaccharides and available carbohydrates. Anal Bioanal Chem 389, 291-308.

44. Björck I, Eliasson A, Drews A, et al. (1990) Some nutritional properties of starch and dietary fiber in barley genotypes containing different levels of amylose. Cereal Chem 67, $327-333$.

45. Ames N, Sopiwnyk E \& Therrien M (2003) Method of Preparing Tortillas from Waxy Barley Cultivars. 426/549;426/
94;426/138;426/808 ed. (Her Majesty the Queen in right of Canada, as represented by the Department of Agriculture \& Agri-Food Canada, editor). United States: A21D 13/00

46. AACC International (1999) Method 44-15.02. Moistureair-oven methods. In Approved Methods of Analysis. St Paul, MN: AACC International.

47. Greenberg DC \& Whitmore ET (1974) A rapid method for estimating the viscosity of barley extracts. J Ins Brew 90, 178-180.

48. Brouns F, Bjorck I, Frayn K, et al. (2005) Glycaemic index methodology. Nutr Res Rev 18, 145-171.

49. Mäkeläinen H, Anttila H, Sihvonen J, et al. (2007) The effect of beta-glucan on the glycemic and insulin index. Eur J Clin Nutr 61, 779-785.

50. Trinidad TP, Mallillin AC, Encabo RR, et al. (2013) The effect of apparent amylose content and dietary fibre on the glycemic response of different varieties of cooked milled and brown rice. Int J Food Sci Nutr 64, 89-93.

51. Granfeldt Y, Drews A \& Bjorck I (1995) Arepas made from high amylose corn flour produce favorably low glucose and insulin responses in healthy humans. $J$ Nutr 125, 459-465.

52. Hallström E, Sestili F, Lafiandra D, et al. (2011) A novel wheat variety with elevated content of amylose increases resistant starch formation and may beneficially influence glycaemia in healthy subjects. Food Nutr Res 55, 7074.

53. Tosh SM (2013) Review of human studies investigating the post-prandial blood-glucose lowering ability of oat and barley food products. Eur I Clin Nutr 67, 310-317.

54. Wood P, Arrigoni E, Miller S, et al. (2002) Fermentability of oat and wheat fractions enriched in beta-glucan using human fecal innoculation. Cereal Chem 79, 445-454.

55. Cummings JH \& Englyst HN (1987) Fermentation in the human large intestine and the available substrates. $\mathrm{Am} \mathrm{J}$ Clin Nutr 45, Suppl. 5, 1243-1255.

56. Battilana P, Ornstein K, Minehira K, et al. (2001) Mechanisms of action of beta-glucan in postprandial glucose metabolism in healthy men. Eur J Clin Nutr 55, 327-333.

57. de Munter JS, Hu FB, Spiegelman D, et al. (2007) Whole grain, bran, and germ intake and risk of type 2 diabetes: a prospective cohort study and systematic review. PLoS Med 4, e261.

58. Samra RA \& Anderson GH (2007) Insoluble cereal fiber reduces appetite and short-term food intake and glycemic response to food consumed $75 \mathrm{~min}$ later by healthy men Am J Clin Nutr 86, 972-979.

59. Bourdon I, Yokoyama W, Davis P, et al. (1999) Postprandial lipid, glucose, insulin, and cholecystokinin responses in men fed barley pasta enriched with $\beta$-glucan. Am J Clin Nutr 69 , $55-63$ 